\title{
The transducer-like protein Tlp12 of Campylobacter jejuni is involved in glutamate and pyruvate chemotaxis
}

\author{
Anastasia-Lisa Lübke', Sabrina Minatelli ${ }^{1}$, Thomas Riedel ${ }^{2,3}$, Raimond Lugert ${ }^{1}$, Isabel Schober ${ }^{2}$, Cathrin Spröer ${ }^{2}$, \\ Jörg Overmann ${ }^{2,3}$, Uwe Groß ${ }^{1}$, Andreas E. Zautner ${ }^{1+}$ and Wolfgang Bohne ${ }^{1 *+}$ (i)
}

\begin{abstract}
Background: Campylobacter jejuni is one of the most common bacterial causes of food-borne enteritis worldwide. Chemotaxis in C. jejuni is known to be critical for the successful colonization of the host and key for the adaptation of the microbial species to different host environments. In C. jejuni, chemotaxis is regulated by a complex interplay of 13 or even more different chemoreceptors, also known as transducer-like proteins (Tlps). Recently, a novel chemoreceptor gene, t/p12, was described and found to be present in $29.5 \%$ of the investigated C. jejuni strains.

Results: In this study, we present a functional analysis of Tlp12 with the aid of a $1 / p 12$ knockout mutant of the $C$. jejuni strain A17. Substrate specificity was investigated by capillary chemotaxis assays and revealed that Tlp 12 plays an important role in chemotaxis towards glutamate and pyruvate. Moreover, the $\Delta t / p 12$ mutant shows increased swarming motility in soft agar assays, an enhanced invasion rate into Caco-2 cells and an increased autoagglutination rate. The growth rate was slightly reduced in the $\Delta t / p 12$ mutant. The identified phenotypes were in partial restored by complementation with the wild type gene. Tlp12-harboring C. jejuni strains display a strong association with chicken, whose excreta are known to contain high glutamate levels.
\end{abstract}

Conclusions: TLP12 is a chemoreceptor for glutamate and pyruvate recognition. Deletion of t/p12 has an influence on distinct physiological features, such as growth rate, swarming motility, autoagglutination and invasiveness.

Keywords: Campylobacter jejuni, Chemoreceptor, Transducer-like protein, Chemotaxis, Glutamate, Pyruvate, Tlp12

\section{Background}

Chemotaxis is a critical mechanism for the successful colonization of host animals and ecological niches by Campylobacter spp. [1, 2]. Their movement towards favorable chemical stimuli such as amino acid carbon sources and away from unfavorable stimuli, e.g. bile acids, drives their movement to niches of optimal growth conditions. Chemotaxis receptors, also referred to as methylaccepting chemotaxis proteins or transducer-like proteins (Tlps), activate flagellar motility by detecting various chemical stimuli in the environment and triggering a signal transduction cascade, which modulates the flagellar motor [3].

\footnotetext{
* Correspondence: wbohne@gwdg.de

${ }^{\dagger}$ Andreas E. Zautner and Wolfgang Bohne contributed equally to this work. 'Institute for Medical Microbiology, University Medical Center, Kreuzbergring 57, 37075 Göttingen, Germany
}

Full list of author information is available at the end of the article
Chemotaxis in C. jejuni is mediated by - according to current knowledge - 13 transducer-like proteins (Tlps) and two aerotaxis proteins (Aer) [4-6]. These chemoreceptors have been grouped into three subtypes, namely A, B and C [5]. Group-A Tlps are integral membrane proteins consisting of two transmembrane domains, an $N$-terminal, periplasmic, sensory domain that binds chemotaxis ligands and a cytoplasmic signaling domain. Tlps in this group are Tlp1, Tlp2, Tlp3, Tlp4, Tlp7, Tlp10, Tlp11, Tl12, and Tlp13. The presence of a periplasmic ligand-binding domain allows the group A Tlps to sense ligands external to the cell. Tlp9 (CetA) is the only group B Tlp. It consists of an $\mathrm{N}$-terminal transmembrane domain anchoring the receptor in the inner membrane of the bacterium and a $C$-terminal cytoplasmic signaling region. It interacts with two cytoplasmic ligand proteins to sense aerotactic signals (Aer1 and 
Aer2) [6]. The group C Tlps include Tlp5, Tlp6 and Tlp8 and are cytoplasmic proteins that detect cytosolic signals [5]. All Tlps are thought to form homodimers and arrange in groups of three [5].

Even though the identification of specific chemoeffectors for the individual Tlps is often complicated due to the frequent compensation of the function of one chemoreceptor for another [7], the specificities of a few TLPs have successfully been identified. Aspartate was found to be the chemoattractant of Tlp1, Tlp3, Tlp6 and Tlp10 [8-10]. Tlp6 and Tlp10 have also been found to be chemoreceptors for glutamine and/or glutamate and TCA cycle intermediates [8]. Formic acid has been identified as chemoattractant of Tlp7 [11, 12]. Tlp3 has been identified as a chemoreceptor for multiple chemoeffectors, therefore named "Campylobacter chemoreceptor for multiple ligands" (CcmL) [10]. Bile acids are usually chemorepellents and are sensed by Tlp3 and Tlp4 [13].

A study by Mund et al. [14] revealed that tlp1, tlp2, tlp3, tlp6, tlp8, tlp9 and tlp10 were all conserved, whereas the $t l p 4, t l p 5, t l p 7, t l p 11, t l p 12$, and $t l p 13$ genes were significantly variable. The tlp4 gene (cj0262c) in the C. jejuni strain NCTC 11168 is flanked by a gene encoding a hypothetical protein (cj0261c) and zupT (cj0263c). Several other $C$. jejuni strains possess at the same position a $t l p$ gene that displays a significantly different sensory domain sequence compared to the $C$. jejuni NCTC 11168 tlp4 gene. In the C. jejuni R14 strain for example, $173 \mathrm{bp}$ of the $5^{\prime}$ end and $1136 \mathrm{bp}$ of the $3^{\prime}$ end of this tlp (H730_01610) were highly similar to the tlp4 gene in NCTC 11168, however, the 682 bp sequence in between shared no significant sequence identity [14]. Thus, the C. jejuni R14 gene (H730_01610), was hypothesized to have a different function to $t l p 4$ and homologs of this gene were named tlp12 [14]. The tlp12 gene is not ubiquitous among C. jejuni strains. From 292 analyzed $C$. jejuni isolates, $29.5 \%$ were found to possess the tlp12 gene [14].

In this study, we performed a functional analysis of Tlp12 with the aid of a $\Delta$ tlp 12 mutant generated in a strain that naturally lacks $t l p 4$, $t l p 11$, and $t l p 13$, as demonstrated by single-molecule real-time (SMRT) genome sequencing. We systematically investigated the chemotactic behavior of this mutant to a variety of compounds that were previously shown to be involved in C. jejuni chemotaxis and could reveal that pyruvate and glutamate are chemoattractors for Tlp12. Moreover, secondary roles of Tlp12 for motility, autoagglutination and biofilm formation were elucidated.

\section{Results}

For functional tlp12 analysis, we chose the $C$. jejuni human stool isolate A17, which contains tlp12, but lacks tlp4, tlp11, and tlp13. Tlp4 was previously shown to share a smaller $N$-terminal and a larger $C$-terminal region with tlp12 (Mund et al. 2016 [14]) and attempts to delete tlp12 in a tlp4-positive $C$. jejuni strain might lead to incorrect integration of the targeting construct. The presence of tlp12 and absence of tlp4 in the A17 isolate was initially detected by PCR [14] and subsequently confirmed by analysis of its complete genome sequence. The genome of the $C$. jejuni strain A17 consists of a circular chromosome of $1,683,497 \mathrm{bp}$ and an extrachromosomal element of 134,322 bp. The chromosome contains 1660 predicted coding sequences (CDS) with a coding density of 0.986 genes per $\mathrm{kb}$ and an average gene size of $928 \mathrm{bp}, 9$ rRNAs and 44 tRNAs. The G $+\mathrm{C}$ content is $30.42 \%$. The extrachromosomal element encodes 152 CDS with a coding density of 1.13 genes per $\mathrm{kb}$ and an average gene size of $772 \mathrm{bp}$.

The coding region of the A17 tlp12 gene predicts a protein of 662 amino acids with a molecular weight of $73.2 \mathrm{kDa}$. The putative domain structure and an alignment with Tlp12 of strain R14 (H730_01610) and Tlp4 of strain NCTC 11168 (Cj0262c) are shown in Additional file 1. A neighbor-joining tree was constructed with the nucleotide sequences of the top thirteen results of a BLAST using the A17 tlp 12 CDS as a query. For comparison, reference sequences of the five $t l p$ genes that are most similar to tlp12 of strain A17 (tlp2, tlp3, tlp4, tlp11 and tlp13 of NCTC 11168) were included in the neighbor-joining tree. The neighbor-joining tree indicates that $t l p 12 \_A 17$ of strain A17 is most closely related to tlp4 of strain NCTC 11168, among the tlp gene reference genes (Fig. 1). Furthermore, the tree reveals a considerable amount of variability among the tlp12 genes obtained from the strain A17 tlp12 BLAST search. Sub-clades of the $t$ lp12 gene can be seen among the fourteen C. jejuni isolates, which include R14, the strain in which the $\operatorname{tlp} 12$ gene was originally identified. A17 tlp12 and R14 tlp12 are seen to be located within different sub-clades. Whether sub-clades are associated with functional differences between the distinct $t l p 12$ alleles remains to be investigated.

\section{Glutamate and pyruvate are chemoattractors for Tlp12}

A syringe-capillary assay was used to determine the chemotactic response of the wild type A17 isolate towards compounds that have been shown previously to act as chemoattractors in C. jejuni. The collection of tested compounds included the five amino acids serine, glutamine, aspartate, cysteine and glutamate and the four intermediates of the central carbon metabolism fumarate, succinate, lactate and pyruvate. All compounds were used at concentrations of $100 \mathrm{mM}$. A compound was considered as a chemoattractant when relative chemotactic response (RCR)-values were $>1.8$, which was the case for lactate, serine, glutamine, glutamate and pyruvate (Table 1). 


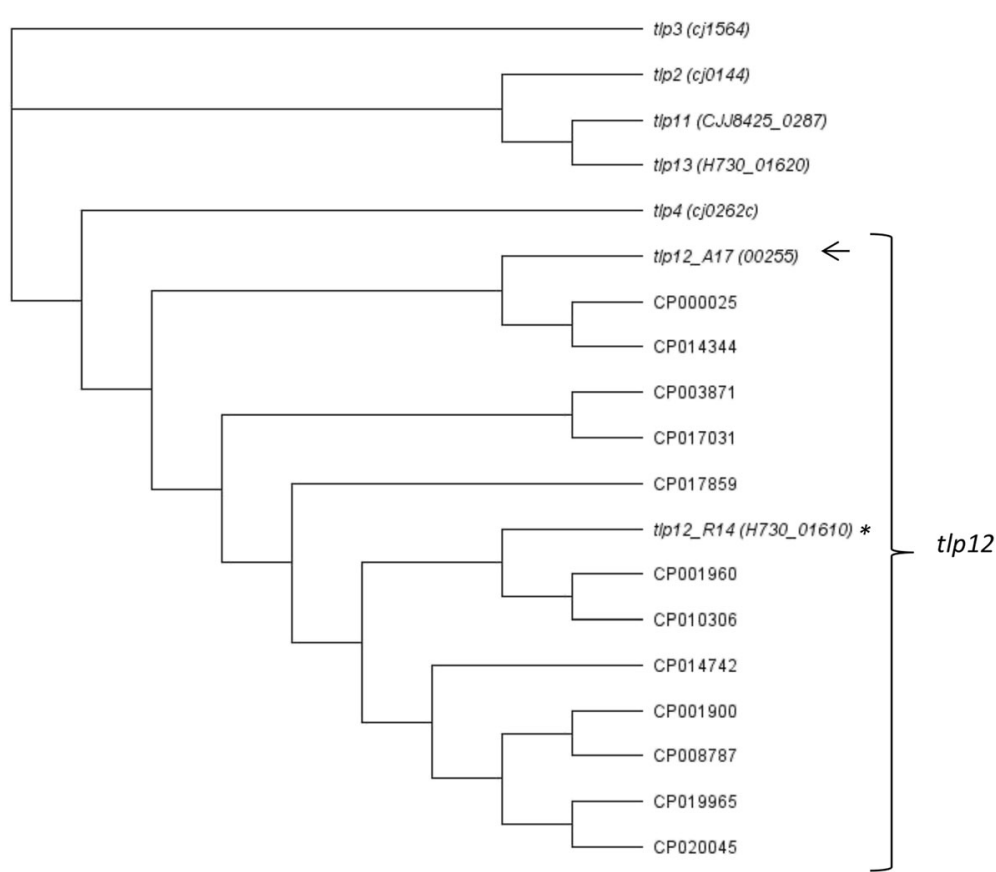

Fig. 1 Neighbor-joining tree. The top thirteen hits of a nucleotide BLAST search using the coding sequence of t/p12 of strain A17 as a query were aligned with the $t / p 12$ sequence of strain A17 together with the reference sequences for $t / p 2$, t/p3, t/p4, t/p 11 and t/p 13 . Global alignment with free end gaps was used. A neighbor-joining tree was constructed using the Geneious 10.0.9 software. The t/p 12 CDS of strainA17 is indicated by an arrow, the t/p12 CDS of strain R14 is indicated by an asterisk

To test the contribution of Tlp12 to chemotaxis towards these compounds, we generated a $\Delta$ tlp12 mutant of strain A17 by integration of a kanamycin cassette via double homologous recombination into the tlp12 locus. Complementation was performed by a plasmid-based, double-recombinational insertion of the tlp12-coding sequence, linked to a chloramphenicol resistance cassette (CamR), into one of the three rRNA loci of the A17 $\Delta$ tlp12 mutant (Additional file 2). Subsequently, syringe-capillary assays were performed in order to compare the chemotaxis of the mutant with the parental strain A17. The $\Delta t l p 12$ mutant showed a significant $(p=$ $0.0044)$ reduction of chemotaxis to glutamate $(13 \%$ of

Table 1 Chemotactic response

\begin{tabular}{lll}
\hline Compound & WT RCR & $\Delta$ t/p12 RCR \\
\hline Aspartate & $0.97 \pm 0.01$ & $0.28 \pm 0.03$ \\
Cysteine & $0.06 \pm 0.03$ & $0.04 \pm 0.01$ \\
Fumarate & $0.68 \pm 0.27$ & $0.70 \pm 0.05$ \\
Glutamate & $18.10 \pm 5.60$ & $2.33 \pm 1.38$ \\
Glutamine & $1.80 \pm 0.01$ & $2.80 \pm 0.16$ \\
Lactate & $3.50 \pm 0.45$ & $6.30 \pm 0.26$ \\
Pyruvate & $7.90 \pm 0.18$ & $1.83 \pm 0.12$ \\
Serine & $2.01 \pm 0.31$ & $1.47 \pm 0.01$ \\
Succinate & $0.50 \pm 0.17$ & $1.53 \pm 0.14$ \\
\hline
\end{tabular}

wild type level), which was partially restored in the complemented mutant (Fig. 2), suggesting that Tlp12 seems to play a major role in glutamate chemotaxis. Pyruvate chemotaxis was reduced in the $\Delta t l p 12$ mutant to $23 \%$ of wild type level, however, $p$-values for pyruvate chemotaxis failed to show statistical significance $(p>0.05)$.

The $\Delta$ t/p12 mutant shows increased swarming motility, invasion and autoagglutination, but a decreased growth rate

The influence of tlp12 deletion on the swarming motility of the mutant was measured in a soft agar assay. The diameter of the motility zone produced by the $\Delta t l p 12$ mutant was 1.7-fold higher than that of the A17 wild type strain and was reduced almost completely back to wild type level after complementation (Fig. 3). We thus conclude that loss of Tlp12 results in an increased swarming motility in the A17 isolate. The invasion rate of the $\Delta t l p 12$ mutant into Caco-2 cells was found to be 4-fold increased and could also be reduced to almost parental strain level after complementation (Fig. 4). In contrast, adhesion to Caco-2 cells was not affected by tlp12 deletion (data not shown). In addition, the $\Delta t \operatorname{tp} 12$ mutant displayed unaltered biofilm formation (data not shown). Interestingly the autoagglutination level was 1.7 -fold increased in the $\Delta t l p 12$ mutant and was restored to almost parental strain level after complementation 
(Fig. 5). Growth experiments revealed that the duration of the lag phase and the duration of the exponential phase of the $\Delta t l p 12$ mutant were unaffected. However, the peak OD values of the $\Delta t l p 12$ mutant were significantly reduced compared to the parental strain (Fig. 6). This reduced growth rate suggests a direct or indirect effect of Tlp12 expression on C. jejuni fitness.

\section{Discussion}

The chemosensitivity of bacteria depends on the repertoire of chemoreceptors expressed by the bacteria, the sensitivity and specificity of the chemoreceptors toward ligands, and on the interplay between different chemoreceptors, which were shown to cooperate in clusters [15]. In this work, the role of the recently identified type A $C$. jejuni chemoreceptor, Tlp12, was studied with the aid of a tlp12 gene deletion mutant. The $\Delta t$ tp12 mutant displayed decreased chemotaxis towards glutamate and pyruvate and was shown to be affected in motility, invasion, growth rate and autoagglutination.

Glutamate has long been known as a chemoattractant for $C$. jejuni $[1,16]$ and plays a vital role in the $C$. jejuni metabolism. Firstly, glutamate can be transaminated to aspartate by the reaction of glutamate and oxaloacetate to produce aspartate and 2-oxoglutarate. This is catalyzed by

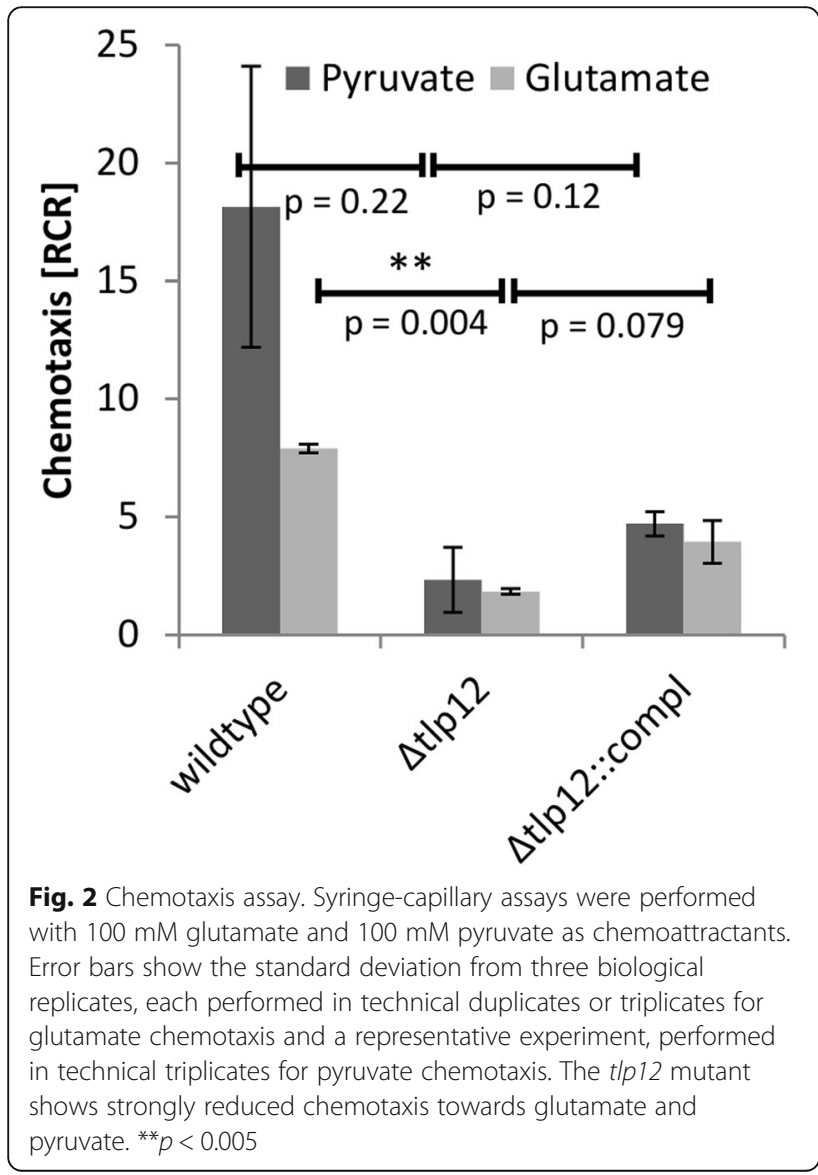

the aminotransferase, AspB, in a reversible reaction [17]. 2-Oxoglutarate may then feed into the TCA cycle to produce ATP, while aspartate is deaminated by the aspartase, AspA, into fumarate, which in turn is also fed into the TCA cycle [17]. Secondly, glutamate can react with free ammonia, producing glutamine in an ATP-dependent manner by the action of glutamine synthetase, GlnA [18]. Thus, glutamate provides the link between nitrogen and carbon metabolism and presents the means for nitrogen assimilation in $C$. jejuni. Both genes, aspB and $g \ln A$, are present in the wild type A17 strain (A17_00816; A17_00757).

Glutamate chemotaxis might be particularly important for the colonization of the chicken gut, since glutamate was shown to be present at very high concentrations in chicken excreta [19, 20]. Chickens are natural hosts for C. jejuni and contaminated chicken meat is believed to be a major source for human campylobacteriosis [21]. The predominant colonization site of $C$. jejuni in the chicken gastrointestinal tract is the cecum, where they are present at $10^{6}-10^{8} \mathrm{CFU} / \mathrm{g}$ of cecal content [22]. C. jejuni isolates differ tremendously in their potential to colonize the chicken gut $[23,24]$ and successful colonization was shown to be a multifactorial process in which a plethora of colonization factors is involved, including chemotaxis genes [25]. Mutants that are deficient

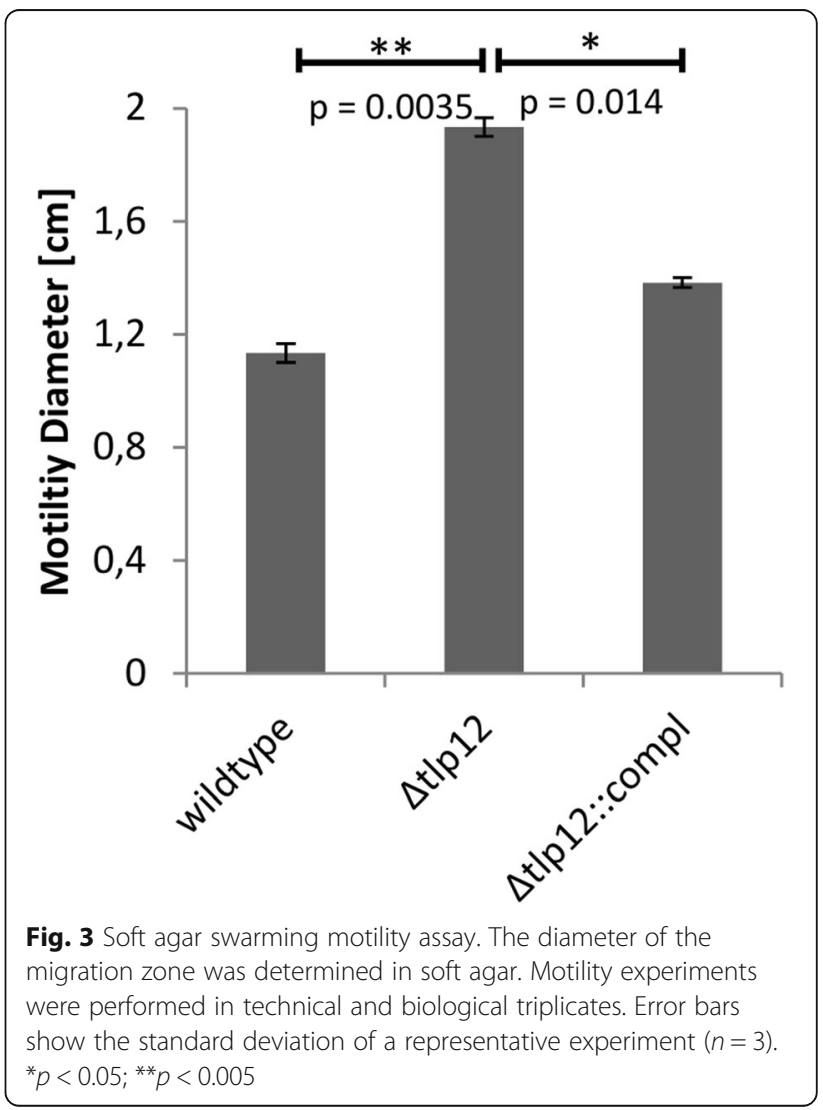


in chemotaxis are no longer able to competitively colonize the chicken gastrointestinal tract [2]. Individual chemoreceptors that were demonstrated to have an effect on chicken gut colonization are Tlp1, Tlp4 and Tlp10 [9, 26]. The presence of the Tlp12 glutamate receptor in $~ 30 \%$ of the $C$. jejuni isolates might thus be an adaptation to the glutamate- rich environment of the chicken gut and may contribute to colonization of the chicken cecum.

The hypothesis that Tlp12 provides a selective advantage for chicken colonization is strengthened by the fact that the frequency of tlp12-positive strains isolated from chicken vs. cattle differs largely. Mund et al. determined the prevalence of the six non-ubiquitous chemoreceptor genes tlp4, tlp5, tlp7, tlp11, tlp12 and tlp13 in C. jejuni isolates of chicken, turkey, bovine and humans [14]. The occurrences of each of the six tlp genes in poultry and bovine were normalized for identical sample numbers and expressed as poultry-occurrence-to-bovine-occurrence (poultry/bovine) ratios, in order to identify putative associations of the $t l p$ genes with either poultry or cattle (Additional file 3). Tlp4, tlp5, tlp 7 and $t l p 11$ were found to be more highly associated with cattle, displaying poultry/bovine ratios of $0.67,0.67,0.16$ and 0.14 , respectively. In contrast, $t l p 12$ and $t l p 13$ showed a clear association with poultry, with poultry/bovine ratios of 2.7 and 10.2 , respectively.

In addition to glutamate, Tlp12 is also involved in pyruvate recognition. Tlps that respond to pyruvate were

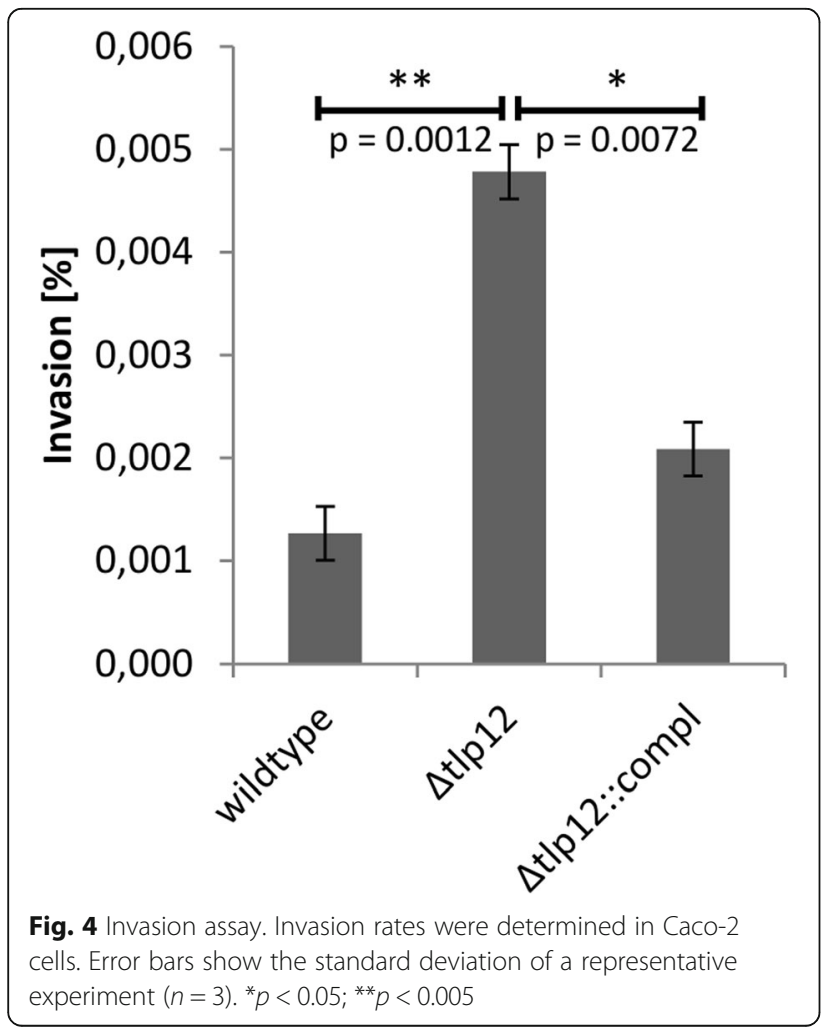

identified before and include Tlp6, Tlp8, Tlp9 and Tlp10 $[7,8]$. This confirms the general known concept that compound recognition in C. jejuni is redundant and that a single ligand can be recognized by more than one chemoreceptor $[8,10]$, which provides robustness to the C. jejuni chemotaxis system.

The $\Delta t$ tp 12 mutant was shown to have an increased swarming motility in soft agar. A similar phenotype was described before for two independently generated $C$. jejuni $\Delta t l p 8$ mutants $[8,27]$ and it was speculated in one of these studies [8], that this phenotype might be due to increased CheY phosphorylation. $\mathrm{CheY}$ is the transducer that regulates the flagellar motor. In $E$. coli, phosphorylated $\mathrm{CheY}$ reverses the flagellar rotation from run to tumbling and an increased tumbling frequency was shown to lead to a larger swarming zone in soft agar, although a "tumbling only" phenotype has a decreased swarming zone [28]. A CheY null mutant in C. jejuni can swim in liquid but fails to develop a swarming zone in soft agar, most likely due to the lack of tumbling [29]. CheY overexpression by a $C$. jejuni diploid $c h e Y$ strain leads to an increased swarming zone [30]. These observations suggest, that as in $E$. coli, the $C$. jejuni phosphoCheY reverses the flagellar rotation from run to tumbling. We thus hypothesize that deletion of tlp12 might change the interaction of the remaining chemoreceptors with proteins involved in the signal transmission (i.e. $\mathrm{CheV}, \mathrm{CheA}$ ) in a manner that finally results in increased

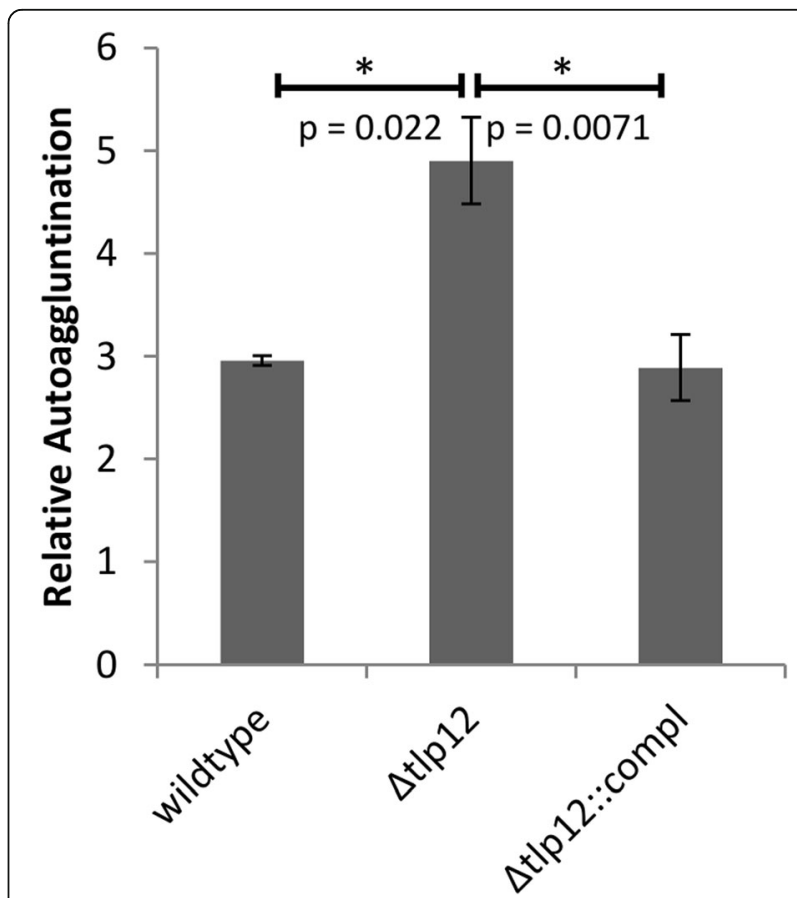

Fig. 5 Autoagglutination assay. The experiments were performed in technical duplicates and in biological triplicates. Error bars show the standard deviation of a representative experiment $(n=3) .{ }^{*} p<0.05$ 


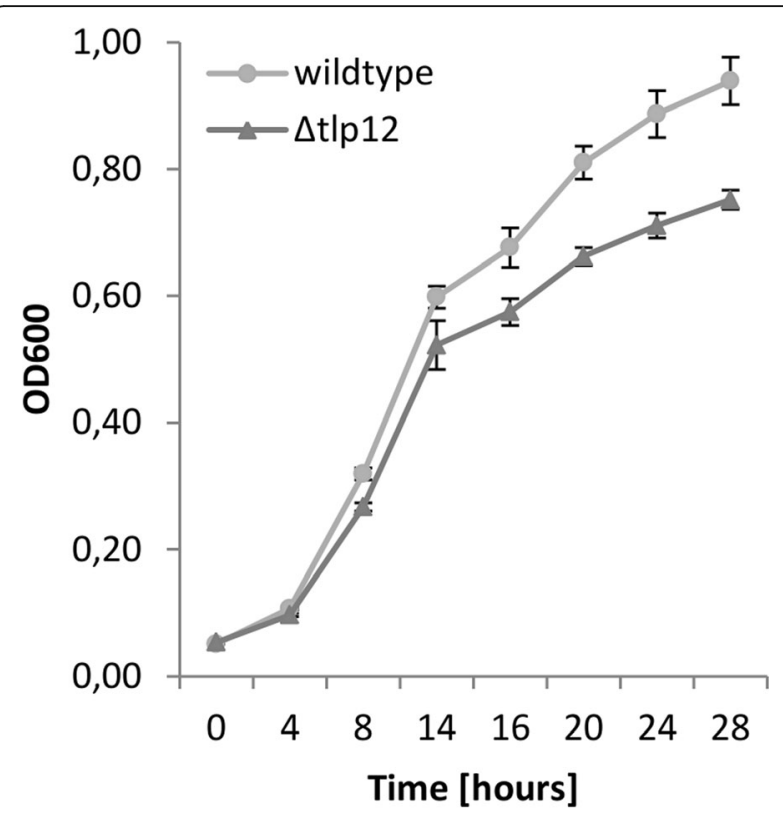

Fig. 6 Growth curve. The growth experiments were performed in biological duplicates or triplicates. Error bars show the standard deviation of a representative experiment. $p$-values were calculated for each time point. A significant difference $(p<0.05)$ between wild type and tlp12 mutant was observed for the time points $16 \mathrm{~h}, 20 \mathrm{~h}$, $24 \mathrm{~h}$ and $28 \mathrm{~h}$.

levels of phospho-CheY and thus in a larger swarming zone.

The $\Delta$ tlp12 mutant also displayed a higher autoagglutination rate, a phenotype that was previously observed for a mutant of the multi-ligand binding chemoreceptor, Tlp3, in NCTC 11168 [10]. However, in contrast to the $\Delta$ tlp3 mutant, no increased biofilm formation ability was detected in the $\Delta$ tlp12 mutant.

\section{Conclusions}

The complex and diverging alterations of phenotypic characteristics observed here for Tlp12, but also for previously investigated tlp mutants, highlights the complex interaction of the $C$. jejuni chemotaxis system with various other cellular systems. A balanced expression and activity pattern of individual tlps appears to be of high importance for adaptive processes in the C. jejuni life cycle.

\section{Methods}

\section{Bacterial culture conditions and cultivation of eukaryotic cells}

Bacteria were maintained at $42{ }^{\circ} \mathrm{C}$ on Columbia agar plates supplemented with sheep blood (COS) in anaerobe containers with a microaerophilic atmosphere, consisting of $5 \% \mathrm{O}_{2}, 10 \% \mathrm{CO}_{2}$ and $85 \% \mathrm{~N}_{2}$ (CampyGen, ThermoScientific). Human colon carcinoma (Caco2) cells were maintained in $75 \mathrm{~cm}^{2}$ cell culture flasks in
$30 \mathrm{ml}$ of Dulbecco Minimal Essential Medium (DMEM) supplemented with $10 \%$ heat inactivated Fetal Calf Serum (FCS), $1 \times$ Non-Essential Amino acids (NEA) and $100 \mathrm{U} / \mathrm{ml}$ penicillin and $100 \mu \mathrm{g} / \mathrm{ml}$ streptomycin.

\section{Growth curves}

Bacterial cultures were prepared for growth experiments by firstly growing pre-cultures of the strains in $20 \mathrm{ml}$ Mueller Hinton (MH) medium in $100 \mathrm{ml}$ Erlenmeyer flasks for $24 \mathrm{~h}$ at $37{ }^{\circ} \mathrm{C}$ and 150 rotations per minute (rpm) under microaerophilic conditions. The ODs were adjusted to $\mathrm{OD}_{600} 0.05$ at the start of the growth experiments by inoculation of $20 \mathrm{ml} \mathrm{MH}$ medium with the prepared pre-cultures. Incubation conditions were as described for the pre-cultures.

\section{Genome sequencing, assembly and annotation}

Genome sequencing was carried out on the PacBio RSII (Pacific Biosciences, Menlo Park, CA) using P6 chemistry. Genome assembly was performed with the "RS_HGAP_Assembly.3" protocol included in SMRT Portal version 2.3.0. The chromosomal contig was trimmed, circularized, and adjusted to dnaA (A17_00001) as first gene. The extrachromosomal element was trimmed and circularized. In addition, genome sequencing of strain A17 was carried out on a NextSeq (Illumina, San Francisco, CA) and quality improvement of the long-read sequence was performed with Burrows-Wheeler Aligner (BWA) [31, 32] mapping the Illumina reads onto the contigs to obtain a final consensus sequence. A final quality of QV60 was confirmed. Automated genome annotation was carried out using Prokka [33]. The complete genome has been deposited at GenBank under accession numbers CP028372 and CP028373.

\section{Swarming motility assays}

Soft agar swarming motility assays were performed as described in [12] with minor modifications. Liquid cultures were grown for $16 \mathrm{~h}$ at $37{ }^{\circ} \mathrm{C}$ under microaerophilic conditions and then adjusted to an $\mathrm{OD}_{600}$ of 0.025 . These cultures were stabbed into $0.4 \% \mathrm{MH}$ agar plates with a $1 \mu \mathrm{l}$ inoculation loop. The plates were then incubated at $37{ }^{\circ} \mathrm{C}$ under microaerophilic conditions for $36 \mathrm{~h}$, after which the diameters of the swarming motility zones were measured.

\section{Eukaryotic cell invasion and adhesion}

Invasion and adhesion assays were performed as described by Everest and coworkers [34] with minor modifications. $\mathrm{CaCo} 2$ cells were seeded in 24-well plates at a concentration of $2 \times 10^{5}$ well. After $16 \mathrm{~h}$ of incubation, $\mathrm{CaCo} 2$ cells were infected with $\sim 2 \times 10^{6} \mathrm{CFU} \mathrm{C}$. jejuni A17 derived mutants, which corresponds to a multiplicity of infection (MOI) of 10. Plates were centrifuged at $600 \mathrm{~g}$ 
for $5 \mathrm{~min}$ and incubated afterwards for two hours at $37^{\circ} \mathrm{C}$ to allow the bacteria to invade the host cells. An aliquot of the bacterial suspension was plated on COS plates in serial dilutions, for a determination of the actual number of CFU added to each well. Following the $2 \mathrm{~h}$ incubation, the bacterial suspension was removed from the $\mathrm{CaCo} 2$ cells and the monolayer was washed three times with $1 \mathrm{ml}$ DMEM before the addition of a $1 \mathrm{ml}$ suspension of $100 \mu \mathrm{g} / \mathrm{ml}$ gentamycin in DMEM, followed by an incubation period of two hours at $37{ }^{\circ} \mathrm{C}$. Afterwards, the cell monolayer was washed again three times with $1 \mathrm{ml}$ of DMEM and lysed with $100 \mu \mathrm{l}$ of $0.1 \%$ Triton X-100 in DMEM to release intracellular bacteria. After $10 \mathrm{~min}$ of incubation, $900 \mu \mathrm{l}$ of DMEM was added and the number of viable bacteria in each well was determined by plating serial dilutions on COS plates. Percentage invasion was calculated by dividing the number of invaded bacteria by the number of viable bacteria that were added to the wells. Adhesion experiments were performed in the same way as described above with the only modification being an infection of the cell monolayer for $30 \mathrm{~min}$ rather than $2 \mathrm{~h}$ and no incubation step with gentamycin. Experiments were performed in technical triplicates and in at least biological duplicates.

\section{Autoagglutination assay}

Autoagglutination assays were carried out as described by Misawa and Blaser [35]. Bacteria grown for $16 \mathrm{~h}$ on COS agar plates at $42{ }^{\circ} \mathrm{C}$ under microaerophilic conditions were resuspended in PBS ( $\mathrm{pH}$ 7.4) and adjusted to an $\mathrm{OD}_{600}$ of 1 . The bacterial suspensions were then added in a volume of $2 \mathrm{ml}$ into glass tubes and incubated for $24 \mathrm{~h}$ at $37^{\circ} \mathrm{C}$ under microaerophilic conditions without shaking. After incubation, $1 \mathrm{ml}$ of the supernatant was carefully removed and the $\mathrm{OD}_{600}$ was measured. Relative autoagglutination was calculated by dividing the initial OD of the bacterial suspension by the final OD of the bacterial supernatant.

\section{Biofilm assay}

The biofilm assays were performed as described by Reeser and coworkers [36] with some modifications. Bacteria grown for $16 \mathrm{~h}$ on COS agar plates were resuspended in $\mathrm{MH}$ medium and adjusted to an $\mathrm{OD}_{600}$ of 0.05. $100 \mu \mathrm{l}$ of the bacterial suspension was then added to each well of a 96-well plate and the plate was incubated for $48 \mathrm{~h}$ at $37{ }^{\circ} \mathrm{C}$ under microaerophilic conditions. Only $\mathrm{MH}$ medium was added to a separate row of wells to serve as the negative control. Following incubation, the bacterial cultures were removed from each well and the plate was dried for $30 \mathrm{~min}$ at $60{ }^{\circ} \mathrm{C} .100 \mu \mathrm{l}$ of $0.1 \%$ crystal violet resuspended in water was then added to each well and left to stain for $15 \mathrm{~min}$ at room temperature. Unbound crystal violet was removed from the wells, the wells were washed two times with $100 \mu \mathrm{l}$ of water and the plate was dried for $15 \mathrm{~min}$ at $60{ }^{\circ} \mathrm{C}$. The bound crystal violet was decolorized by adding $100 \mu \mathrm{l}$ solutions of $80 \%$ ethanol and $20 \%$ acetone into each well for $15 \mathrm{~min}$. After $15 \mathrm{~min}$ of decolorization, $90 \mu \mathrm{l}$ of the bacterial solution was pipetted out of the wells and added to a fresh 96-well plate (as not all of the biofilm might have detached from the wells of the old plate after $15 \mathrm{~min}$ ). The plate was then read at an absorbance of $570 \mathrm{~nm}$ with a microplate reader to quantify the amount of biofilm formed in each well. Results were normalized by subtracting absorbances obtained by the negative controls from these readings. The experiments were done for all strains in technical quadruplets and biological triplicates.

\section{$\Delta$ t/p12 knockout generation}

The $\Delta t l p 12$ knock-out mutant was generated by double homologous recombination that resulted in the insertion of a kanamycin resistance cassette, $k a n R$, into the gene. For the construction of the knockout vector, a $546 \mathrm{bp}$

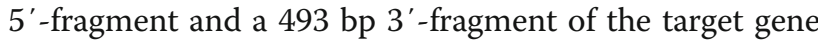
in the $C$. jejuni strain A24 were amplified with the primers psk-5-TLP12-F/kan-5-TLP12-R and kan-3-TLP12-F/psk3-TLP12-R, respectively. The psk-5-TLP12-F and psk3-TLP12-R primers have $30 \mathrm{bp}$ of oligonucleotides that are complementary to the pBluescript SKII vector attached to their $5^{\prime}$ and $3^{\prime}$ ends, respectively. The kan3-TLP12-F and kan-5-TLP12-R primers have 30 bp of oligonucleotides that are complementary to the ends of the kanR gene attached to their $5^{\prime}$ and $3^{\prime}$ ends, respectively. The kanR gene was amplified using the Kan 1 and Kan 2 primers. The PCRs were performed as follows: $95{ }^{\circ} \mathrm{C}$ for $1 \mathrm{~min}, 35$ cycles at $98^{\circ} \mathrm{C}$ for $15 \mathrm{~s}, 58{ }^{\circ} \mathrm{C}$ for $15 \mathrm{~s}$ and $72{ }^{\circ} \mathrm{C}$ for $1 \mathrm{~min}$ and a final incubation at $72{ }^{\circ} \mathrm{C}$ for $5 \mathrm{~min}$. The PCRBio HiFi Kit was used for all PCRs and PCR reactions were put together as per manufacturer's recommendations with $1 \mathrm{U}$ of PCRBIO HIFI polymerase, $400 \mathrm{nM}$ forward and reverse primers and $\sim 500 \mathrm{ng}$ of genomic template DNA in $50 \mu \mathrm{l}$ reactions. The pBluescript SKII vector was digested with BamHI and EcoRI to linearize the vector. The linearized vector, the $3^{\prime}$ and $5^{\prime}$ target gene fragments and the $k a n R$ gene were assembled using the NEBuilder HiFi DNA Assembly Cloning Kit (NEB) and the assembled knockout vector was transformed into NEB 5-alpha competent E. coli. Plasmids were extracted from single colonies and the correct construction of the knockout vector was confirmed by Sanger sequencing using the psk-5-TLP12-F and psk-3-TLP12-R primers. The knockout vector was electroporated into C. jejuni A17 wild type competent cells using an ECM600 Electro Cell Manipulator (BTX) with the following settings: resistance $-2.5 \mathrm{kV}$, capacitance timing - $25 \mu \mathrm{F}$ and resistance timing $186 \Omega$. After electroporation, $100 \mu \mathrm{l}$ SOC Outgrowth 
Medium (NEB) was added to the mixture which was then inoculated onto COS plates and incubated for $16 \mathrm{~h}$ at $37{ }^{\circ} \mathrm{C}$ under microaerophilic conditions to encourage the amplification of the generated mutants. Wild type strains in the background were then excluded by replating the bacteria onto COS plates containing kanamycin $(10 \mu \mathrm{g} / \mathrm{ml})$. Primer sequences are listed in Additional file 4.

\section{$\Delta$ tIp12 complementation}

The complementation of the $\Delta t l p 12$ mutation was performed as described in [37] with some modifications. The complementation was performed by a plasmidbased, double-recombinational insertion of the tlp12-coding sequence linked to a chloramphenicol resistance cassette $(c a m R)$ into one of the three rRNA loci of the A17 $\Delta t l p 12$ mutant. For the construction of the tlp12 complementation vector, the tlp12 gene (A17_00255) from the wild type A17 genome was amplified using the TLP12_Complement Primer_Fw and TLP12_Complement Primer_Rev. At its $5^{\prime}$ end, the TLP12_Complement Primer_Fw has a Shine-Dalgarno sequence for improved binding of RNA polymerase after transcription of the gene and $36 \mathrm{bp}$ of an oligonucleotides sequence that is complementary to the linearized pRRC vector at its $5^{\prime}$ end. The TLP12_Complement Primer_Rev primer has 36 bp of oligonucleotides that are complementary to the linearized pRRC vector at its $3^{\prime}$ end. The tlp12 gene was amplified as follows: $95{ }^{\circ} \mathrm{C}$ for $1 \mathrm{~min}, 35$ cycles at $95{ }^{\circ} \mathrm{C}$ for $15 \mathrm{~s}, 65^{\circ} \mathrm{C}$ for $15 \mathrm{~s}$ and $72{ }^{\circ} \mathrm{C}$ for $2 \mathrm{~min}$ and a final incubation at $72{ }^{\circ} \mathrm{C}$ for $5 \mathrm{~min}$. The PCR reactions were put together as described previously with the PCRBio HiFi Kit. The pRRC vector was digested with $\mathrm{XbaI}$ and phosphatase treated with Antarctic Phosphatase (NEB), as per manufacturer's recommendations. The linearized vector and the tlp12 gene were assembled and transformed into NEB 5-alpha competent E. coli as described previously. The correct construction of the knockout vector was confirmed by Sanger sequencing. Electroporation of the complementation vector into A17 $\Delta$ tlp 12 was performed as described before. The selective chloramphenicol concentration was $12.5 \mu \mathrm{g} / \mathrm{ml}$. The complementation mutant was named $\Delta t l p 12::$ compl. The used primer sequences are listed in Additional file 4.

\section{Chemotaxis syringe capillary assay}

The chemotaxis assays were performed as described by Chandrashehkar et al. [8]. All chemicals tested in the chemotaxis assays were suspended in PBS, adjusted to a $\mathrm{pH}$ of 7 and set to a concentration of $100 \mathrm{mM}$, a concentration that has previously been shown to result in the strongest chemotaxis response [7, 12]. Bacteria grown for $18 \mathrm{~h}$ on $\mathrm{COS}$ agar plates at $42{ }^{\circ} \mathrm{C}$ under microaerophilic conditions were resuspended in $1 \mathrm{ml}$ of PBS (pH 7) and washed once by centrifuging at $4500 \mathrm{~g}$ for $5 \mathrm{~min}$. The washed bacterial suspension was then adjusted to an $\mathrm{OD}_{600}$ of $0.5 .100 \mu \mathrm{l}$ of the tested chemical solutions were drawn through a $27 \mathrm{G}$ hypodermic needle $(0.40 \mathrm{~mm}$ diameter $\times 20 \mathrm{~mm}$ long) into a $1 \mathrm{ml}$ Luer syringe. Buffer alone served as a control. A $100 \mu \mathrm{l}$ of the adjusted bacterial suspension was then drawn into a $200 \mu \mathrm{l}$ disposable pipette and the tip was sealed with parafilm. The pipette tip was attached to the needle-syringe system such that most of the needle immersed into the bacterial suspension. The whole system was then incubated horizontally for $1 \mathrm{~h}$ at $42{ }^{\circ} \mathrm{C}$ under microaerophilic conditions, after which the needle-pipette tip system was detached from the syringe. The bacterial suspension in the syringe was then plated in 10 -fold serial dilutions on COS plates for $48 \mathrm{~h}$ at $37^{\circ} \mathrm{C}$ or $42{ }^{\circ} \mathrm{C}$ under microaerophilic conditions and the $\mathrm{CFU}$ were counted. The taxis toward a test compound was expressed by calculating the Relative Chemotaxis Ratio (RCR), which is the ratio of the number of bacteria in the syringe with a tested chemical to the number in the control (buffer only) after $1 \mathrm{~h}$ incubation. Capillary assays for glutamate and pyruvate were performed at least in biological triplicates, each with at least technical duplicates.

\section{Statistical analysis}

Statistical analysis were performed by two-sided, unpaired Student T-tests using Excel software.

\section{Additional files}

Additional file 1: Amino acid alignment of TLP12 of strain A17, TLP12 of strain R14 (H730_01610) and TLP4 of C. jejuni NCTC 11168 (cj0262C). (JPG $661 \mathrm{~kb})$

Additional file 2: Depicts schematics of the double homologous recombination events that resulted in the deletion of t/p12 and the insertion of the wild type t/p12 gene into one of the three rRNA clusters in the A17 $\Delta$ t/p 12 mutant for the complementation of the mutation. (PNG $340 \mathrm{~kb}$ )

Additional file 3: Distribution of tlps in poultry and cattle. (DOCX $13 \mathrm{~kb}$ ) Additional file 4: Oligonucleotides. (DOCX $18 \mathrm{~kb}$ )

\section{Abbreviations}

BLAST: Basic local alignment search tool; CFU: Colony forming units; COS: Columbia agar + sheep blood; DMEM: Dulbecco Modified Eagle Medium; SMRT: Single-molecule real-time; TLP: Transducer-like protein

\section{Acknowledgements}

We sincerely thank Simone Severitt and Carola Berg from the Leibniz Institute DSMZ for technical assistance.

\section{Funding}

This publication was funded by the Open Access support program of the Deutsche Forschungsgemeinschaft, the publication fund of the Georg August University Göttingen and the German Center for Infection Research (DZIF) (BMBF grant 8000-105-3 to J.O). 


\section{Availability of data and materials}

The datasets used and analyzed within the current study are available from the NCBI website as referenced in the paper. The complete genome has been deposited at GenBank under accession numbers CP028372 and CP028373.

\section{Authors' contributions}

$\mathrm{AL}$ and SM conducted all the experiments apart from the genome sequencing. TR, IS and CS performed C. jejuni A17 genome sequencing and annotation. WB and AZ conceived and coordinated the study and wrote major parts of the manuscript. UG, JO and RL were involved in data interpretation and revising of the manuscript. All authors have read and approved the manuscript.

\section{Consent for publication}

Not applicable.

\section{Competing interests}

The authors declare that they have no competing interests.

\section{Publisher's Note}

Springer Nature remains neutral with regard to jurisdictional claims in published maps and institutional affiliations.

\section{Author details}

${ }^{1}$ Institute for Medical Microbiology, University Medical Center, Kreuzbergring 57, 37075 Göttingen, Germany. 'Leibniz Institute DSMZ-German Collection of Microorganisms and Cell Cultures, Braunschweig, Germany. ${ }^{3}$ German Center for Infection Research (DZIF), Partner Site Hannover-Braunschweig, Braunschweig, Germany.

Received: 25 June 2018 Accepted: 28 August 2018

Published online: 10 September 2018

\section{References}

1. Hugdahl MB, Beery JT, Doyle MP. Chemotactic behavior of Campylobacter jejuni. Infect Immun. 1988;56(6):1560-6.

2. Thibodeau A, Fravalo P, Taboada EN, Laurent-Lewandowski S, Guevremont E, Quessy S, Letellier A. Extensive characterization of Campylobacter jejun chicken isolates to uncover genes involved in the ability to compete for gut colonization. BMC Microbiol. 2015;15:97.

3. Lertsethtakarn P, Ottemann KM, Hendrixson DR. Motility and chemotaxis in Campylobacter and Helicobacter. Annu Rev Microbiol. 2011;65:389-410.

4. Day CJ, Hartley-Tassell LE, Shewell LK, King RM, Tram G, Day SK, Semchenko EA, Korolik V. Variation of chemosensory receptor content of Campylobacter jejuni strains and modulation of receptor gene expression under different in vivo and in vitro growth conditions. BMC Microbiol. 2012;12:128.

5. Marchant J, Wren B, Ketley J. Exploiting genome sequence: predictions for mechanisms of Campylobacter chemotaxis. Trends Microbiol. 2002;10(4): 155-9.

6. Zautner AE, Tareen AM, Gross U, Lugert R. Chemotaxis in Campylobacter jejuni. Eur J Microbiol Immunol (Bp). 2012;2(1):24-31.

7. Vegge CS, Brondsted L, Li YP, Bang DD, Ingmer $\mathrm{H}$. Energy taxis drives Campylobacter jejuni toward the most favorable conditions for growth. Appl Environ Microbiol. 2009;75(16):5308-14.

8. Chandrashekhar K, Gangaiah D, Pina-Mimbela R, Kassem II, Jeon BH, Rajashekara G. Transducer like proteins of Campylobacter jejuni 81-176: role in chemotaxis and colonization of the chicken gastrointestinal tract. Front Cell Infect Microbiol. 2015;5:46.

9. Hartley-Tassell LE, Shewell LK, Day CJ, Wilson JC, Sandhu R, Ketley JM, Korolik $\mathrm{V}$. Identification and characterization of the aspartate chemosensory receptor of Campylobacter jejuni. Mol Microbiol. 2010;75(3):710-30.

10. Rahman H, King RM, Shewell LK, Semchenko EA, Hartley-Tassell LE, Wilson JC, Day CJ, Korolik V. Characterisation of a multi-ligand binding chemoreceptor CcmL (Tlp3) of Campylobacter jejuni. PLoS Pathog. 2014; 10(1):e1003822.

11. Kassem II, Khatri M, Esseili MA, Sanad YM, Saif YM, Olson JW, Rajashekara G. Respiratory proteins contribute differentially to Campylobacter jejuni's survival and in vitro interaction with hosts' intestinal cells. BMC Microbiol. 2012;12:258
12. Tareen AM, Dasti Jl, Zautner AE, Gross U, Lugert R. Campylobacter jejuni proteins Cj0952c and Cj0951c affect chemotactic behaviour towards formic acid and are important for invasion of host cells. Microbiology. 2010;156(Pt 10):3123-35.

13. Li Z, Lou H, Ojcius DM, Sun A, Sun D, Zhao J, Lin X, Yan J. Methyl-accepting chemotaxis proteins 3 and 4 are responsible for Campylobacter jejuni chemotaxis and jejuna colonization in mice in response to sodium deoxycholate. J Med Microbiol. 2014;63(Pt 3):343-54.

14. Mund NL, Masanta WO, Goldschmidt AM, Lugert R, Gross U, Zautner AE. Association of Campylobacter Jejuni ssp. Jejuni chemotaxis receptor genes with multilocus sequence types and source of isolation. Eur J Microbiol Immunol (Bp). 2016;6(3):162-77.

15. Bray D, Levin MD, Morton-Firth CJ. Receptor clustering as a cellular mechanism to control sensitivity. Nature. 1998;393(6680):85-8.

16. Chandrashekhar K, Kassem II, Rajashekara G. Campylobacter jejuni transducer like proteins: chemotaxis and beyond. Gut Microbes. 2017; 8(4):323-34.

17. Guccione E, Leon-Kempis Mdel R, Pearson BM, Hitchin E, Mulholland F, van Diemen PM, Stevens MP, Kelly DJ. Amino acid-dependent growth of Campylobacter jejuni: key roles for aspartase (AspA) under microaerobic and oxygen-limited conditions and identification of AspB (Cj0762), essential for growth on glutamate. Mol Microbiol. 2008;69(1): 77-93.

18. Stahl M, Butcher J, Stintzi A. Nutrient acquisition and metabolism by Campylobacter jejuni. Front Cell Infect Microbiol. 2012;2:5.

19. Parsons CM, Potter LM, Brown RD Jr. Effects of dietary carbohydrate and of intestinal microflora on excretion of endogenous amino acids by poultry. Poult Sci. 1983:62(3):483-9.

20. Kadim IT, Moughan PJ, Ravindran V. Ileal amino acid digestibility assay for the growing meat chicken--comparison of ileal and excreta amino acid digestibility in the chicken. Br Poult Sci. 2002;43(4):588-97.

21. Eurosurveillance editorial team. The European Union summary report on trends and sources of zoonoses, zoonotic agents and food-borne outbreaks in 2010. Euro Surveill. 2012;17(10):20113. PMID 22433599.

22. Svetoch EA, Stern NJ. Bacteriocins to control Campylobacter spp. in poultry-a review. Poult Sci. 2010:89(8):1763-8.

23. Hanel I, Borrmann E, Muller J, Muller W, Pauly B, Liebler-Tenorio EM, Schulze F. Genomic and phenotypic changes of Campylobacter jejuni strains after passage of the chicken gut. Vet Microbiol. 2009;136(1-2): $121-9$.

24. Ringoir DD, Korolik V. Colonisation phenotype and colonisation potential differences in Campylobacter jejuni strains in chickens before and after passage in vivo. Vet Microbiol. 2003;92(3):225-35.

25. Hermans D, Van Deun K, Martel A, Van Immerseel F, Messens W, HeyndrickX M, Haesebrouck F, Pasmans F. Colonization factors of Campylobacter jejuni in the chicken gut. Vet Res. 2011;42:82.

26. Hendrixson DR, DiRita VJ. Identification of Campylobacter jejuni genes involved in commensal colonization of the chick gastrointestinal tract. Mol Microbiol. 2004:52(2):471-84.

27. Reuter $M$, van Vliet $A H$. Signal balancing by the CetABC and CetZ chemoreceptors controls energy taxis in Campylobacter jejuni. PLoS One. 2013;8(1):e54390.

28. Wolfe AJ, Berg HC. Migration of bacteria in semisolid agar. Proc Natl Acad Sci U S A. 1989;86(18):6973-7.

29. Kanungpean D, Kakuda T, Takai S. False positive responses of Campylobacter jejuni when using the chemical-in-plug chemotaxis assay. J Vet Med Sci. 2011;73(3):389-91.

30. Yao R, Burr DH, Guerry P. CheY-mediated modulation of Campylobacter jejuni virulence. Mol Microbiol. 1997;23(5):1021-31.

31. Li H, Durbin R. Fast and accurate short read alignment with burrowswheeler transform. Bioinformatics. 2009;25(14):1754-60.

32. Li H, Durbin R. Fast and accurate long-read alignment with burrowswheeler transform. Bioinformatics. 2010;26(5):589-95

33. Seemann T. Prokka: rapid prokaryotic genome annotation. Bioinformatics. 2014;30(14):2068-9.

34. Everest PH, Goossens H, Butzler JP, Lloyd D, Knutton S, Ketley JM, Williams PH. Differentiated Caco-2 cells as a model for enteric invasion by Campylobacter jejuni and C. coli. J Med Microbiol. 1992; 37(5):319-25.

35. Misawa N, Blaser MJ. Detection and characterization of autoagglutination activity by Campylobacter jejuni. Infect Immun. 2000;68(11):6168-75. 
36. Reeser RJ, Medler RT, Billington SJ, Jost BH, Joens LA. Characterization of Campylobacter jejuni biofilms under defined growth conditions. Appl Environ Microbiol. 2007;73(6):1908-13.

37. Karlyshev AV, Wren BW. Development and application of an insertional system for gene delivery and expression in Campylobacter jejuni. Appl Environ Microbiol. 2005;71(7):4004-13.

Ready to submit your research? Choose BMC and benefit from:

- fast, convenient online submission

- thorough peer review by experienced researchers in your field

- rapid publication on acceptance

- support for research data, including large and complex data types

- gold Open Access which fosters wider collaboration and increased citations

- maximum visibility for your research: over $100 \mathrm{M}$ website views per year

At $B M C$, research is always in progress.

Learn more biomedcentral.com/submissions 